\title{
Extended Diffuse Radio Emission in Abell 115
}

\author{
Cosmos Dumba* \\ Thüringer Landessternwarte Tautenburg, Sternwarte 5, 07778 Tautenburg, Germany \\ E-mail: dumba@tls-tautenburg.de
}

Matthias Hoeft

Thüringer Landessternwarte Tautenburg, Sternwarte 5, 07778 Tautenburg, Germany

E-mail: hoeftetls-tautenburg.de

\begin{abstract}
Alexander Drabent
Thüringer Landessternwarte Tautenburg, Sternwarte 5, 07778 Tautenburg, Germany

E-mail: alexetls-tautenburg.de
\end{abstract}

\section{Annalisa Bonafede}

Hamburger Sternwarte, Gojenbergsweg 112, 21029 Hamburg, Germany

E-mail: annalisa.bonafede@hs.uni-hamburg.de

\begin{abstract}
Merging galaxy clusters have been found to host large-scale diffuse emission known as relics at their periphery. The relics are proposed to arise from merger shocks in the intracluster medium. $\mathrm{X}$-ray observations in some galaxy clusters hosting radio relics have revealed density jumps associated with shocks. The jumps have been shown to trace the diffuse radio emission morphology. Several recent findings indicate that the origin of radio relics is not as previously thought. For instance, the X-ray Mach number of many shock fronts associated with relics is low, and that relics are possibly related to AGN activity which provides a relativistic electron population that gets re-accelerated at the shock front.

Abell 115 is a massive merging galaxy cluster hosting a large-scale diffuse radio emission structure classified as a relic. Abell 115 was studied at $1.4 \mathrm{GHz}$ and in the X-ray showing that the morphology is atypical of a relic; being significantly extended to the east. A study on the dynamic state of Abell 115 was conducted using 88 spectroscopically confirmed members suggesting that the nature of its sub-cluster components indicated a pre-merger state. For such a scenario, a shock front at the location of the relic would not be plausible. This may question if the diffuse emission in Abell 115 traces a shock front as proposed for radio relics.

We study this galaxy cluster by using archival data from the VLA $(1.4 \mathrm{GHz})$ and data from the GMRT (610 MHz) and WSRT (350 MHz). Images and flux measurements from the new observations are provided. Flux measurements of an additional extended source to the east of the cluster center are also provided.
\end{abstract}

EXTRA-RADSUR2015 (*)

20-23 October 2015

Bologna, Italy

(*) This conference has been organized with the support of the Ministry of Foreign Affairs and International Cooperation, Directorate General for the Country Promotion (Bilateral Grant Agreement ZA14GR02 - Mapping the Universe on the Pathway to SKA)

\footnotetext{
*Speaker.
} 


\section{Introduction}

Galaxy clusters often have a large variety of radio galaxies. Furthermore, some merging clusters show extended diffuse emission without optical counterparts as in the galaxy cluster Abell 115. The extended radio emission in Abell 115 does not have an optical counterpart and has a low surface brightness. Objects like these are usually extended $(\sim 1 \mathrm{Mpc})$ and have a steep spectral index typically steeper than -1 , with $S(v) \propto v^{-\alpha}$, where $v$ is the observed frequency and $S$ the flux density.

Diffusive shock acceleration predicts a power spectrum with a slope of 0.5 or steeper right at the shock front. Including synchrotron and IC losses, we do expect a slope of $1-1.5$.

Abell 115 has been studied from the early 80s by [2] in the X-Ray finding a double morphology in the cluster. The relic was noted to reside in the northern clump. Studying the dynamical status of this cluster through $\mathrm{X}$-Ray, [6] showed that the high temperature in the linking region between the two distinct peaks in the surface brightness distribution implied that Abell 115 is a merger system. Earlier studies by [4] on six galaxy clusters including Abell 115 in the radio and X-ray confirmed the existence of an extended diffuse radio relic.

In this paper, we present findings from three separate observations of Abell 115. Results from two recent observations with the GMRT and WSRT are shown. Archival data from the VLA has also been included to obtain a spectral slope.

\section{Relic Location}

According to many findings, radio relics are found at the cluster periphery while halos at the cluster center [3]. For Abell 115, it is very close to the center with X-ray images showing that both peaks of the sub-clusters merging are not far from the relic. So, is the relic directly in our line of sight? [1] analyzed the two sub-clusters and suggested that the one located south had a higher velocity dispersion indicating it was more massive than the northern sub-cluster. They went further to show how it best described a shock that is perpendicular to this center. The underlying question that remains is whether this distance from the merger axis is enough to produce a shock that would generate such extended diffuse emission. The relic is not symmetric with the merger axis of the two main cluster components. We speculate that this is caused by a large impact parameter. Therefore, although the two original cluster centers have slung around each other, no core passage has occurred so far. To the east of the cluster centroid lies an extended diffuse emission source with no evident optical counterpart.

\section{Radio Image Analyses \& Relic Flux Measurements}

Archival data from the VLA was downloaded and re-reduced to produce the image at $1.5 \mathrm{GHz}$. Data from the GMRT and WSRT was also used to obtain images and flux values at $610 \mathrm{MHz}$ and $350 \mathrm{MHz}$ respectively. All the data reduction and calibration steps were done with the CASA software. The AOFlagger was used to remove RFI before calibration procedures [5]. It should be noted here that the bright double-lobed radio galaxy $3 \mathrm{C} 28$, close to the western edge of the relic, causes severe imaging artefacts that prevent a deeper cleaning. Images obtained from all three data sets and an overlay with X-ray are shown in Figure 1. 

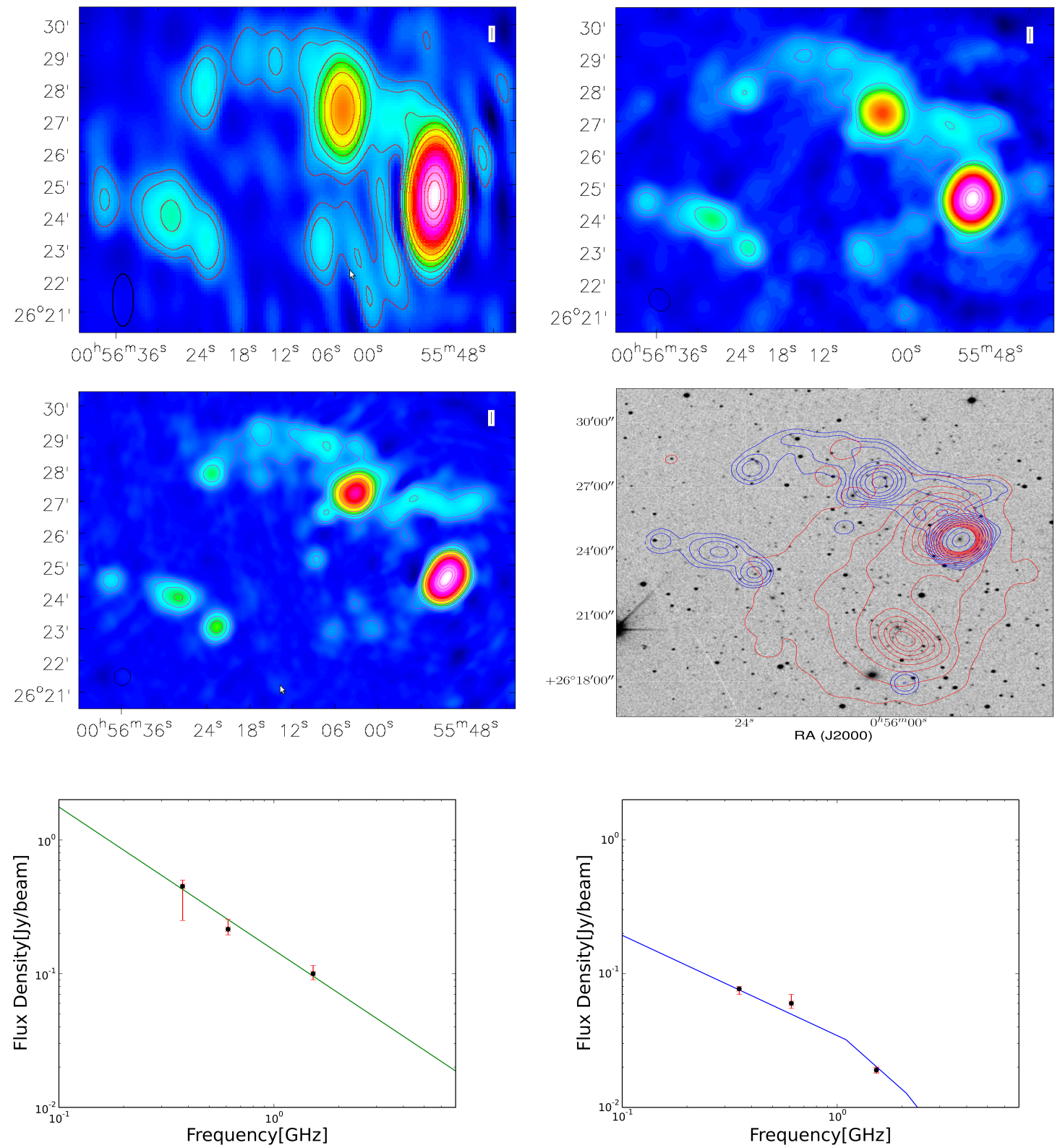

Figure 1: The WSRT at $351 \mathrm{MHz}$ (Top left), GMRT at $612 \mathrm{MHz}$ (Top right) and VLA at $1512 \mathrm{MHz}$ (Middle left) images with contours starting from $3 \times \mathrm{rms}$ are shown. Contour overlays of a VLA image at $1.4 \mathrm{GHz}$ and an X-ray image obtained from the Chandra X-ray Observatory are shown in the middle right image with an Optical background from the DSS. The overlay with X-ray shows the relative position of the main cluster substructures in Abell 115 with the extended diffuse radio emission. The plots at the bottom show flux measurements at the three different frequencies, the relic (bottom left) and the Eastern source (bottom right). The spectral index for the relic is $\sim 1.0$ while the eastern source shows a break in its spectrum from $\sim 0.6$ to $\sim 1.0$. The higher uncertainty in the WSRT $(351 \mathrm{MHz})$ image is partly due to the poorer resolution, thus making it harder to subtract compact sources from the relic region. Flux measurements from the WSRT were difficult due to an overlap of both the NAT galaxy and 3C28. The flux density from a visible region has been extrapolated to the regions covered by the NAT galaxy and $3 \mathrm{C} 28$. 


\section{Discussion}

Data available so far is sufficient for a good estimate of the spectral index, $\alpha \sim 1.0$.

Optical studies that suggested a pre-merger stage iterate the complexity of the dynamics of merging galaxy clusters. The inclination of the merging system is vital in such complex systems since the close proximity of relic emission structures to the cluster center is unlikely.

The analysis is difficult due to the bright radio galaxy $3 \mathrm{C} 28$. With its two extended lobes, an accurate model for subtraction is difficult to determine.

The NAT galaxy located within the relic region obscures the precise measurement of flux underneath. Estimating the extent to which we can subtract it and retain the diffuse radio emission underneath is a challenge. However, with different imaging techniques, high resolution images were able to provide reasonable flux values of all the compact sources. With the flux values of the compact sources in mind, better estimates of how much flux is retained after subtraction were obtained.

\section{Summary}

We were able to measure flux in the relic region for three frequencies; at $1521 \mathrm{MHz}$ (VLA), 610 $\mathrm{MHz}$ (GMRT) and $350 \mathrm{MHz}$ (WSRT). We find a spectral index, $\alpha \sim 1.0$. The relic morphology is consistent at all frequencies. This supports earlier studies that the extended diffuse emission is a relic as found in other galaxy clusters.

The western part of the relic agrees with the northern edge in X-ray surface brightness distribution, suggesting the presence of a shock. The eastern part of the relic remains enigmatic since it extends into a region with very low $\mathrm{X}$-ray surface brightness. We do not find any obvious differences along the relic. However, for a detailed analysis of the spectral index distribution, deeper observations which also allow the removal of $3 \mathrm{C} 28$ without artefacts are necessary.

We find that the eastern diffuse emission source has a curved spectrum, indicating a radio galaxy or fossil plasma.

Acknowledgement: C.D acknowledges funding from the Deutscher Akademischer Austauschdienst.

\section{References}

[1] Barrena, R., Boschin, W., Girardi, M., \& Spolaor, M. 2007, Astronomy \& Astrophysics, 469, 861

[2] Fernelius, N. C. 1981, Journal of Applied Physics, 52, 6285

[3] Giovannini, G., Bonafede, A., Feretti, L., et al. 2009, Astronomy \& Astrophysics, 507, 1257

[4] Govoni, F., Feretti, L., Giovannini, G., et al. 2001, Astronomy \& Astrophysics, 376, 803

[5] Offringa, A. R., van de Gronde, J. J., \& Roerdink, J. B. T. M. 2012, Astronomy \& Astrophysics, 539, A95

[6] Shibata, R., Honda, H., Ishida, M., Ohashi, T., \& Yamashita, K. 1999, The Astrophysical Journal, 524, 603 